\title{
Multiplicité de modèles en Australie
}

\section{Michael Berthold}

\section{(2) OpenEdition}

Journals

Édition électronique

URL : http://journals.openedition.org/ries/3956

DOI : 10.4000/ries.3956

ISSN : 2261-4265

\section{Éditeur}

Centre international d'études pédagogiques

\section{Édition imprimée}

Date de publication : 7 septembre 1995

Pagination : 59-69

ISSN : 1254-4590

\section{Référence électronique}

Michael Berthold, "Multiplicité de modèles en Australie », Revue internationale d'éducation de Sèvres [En ligne], 07 | 1995, mis en ligne le 02 octobre 2014, consulté le 01 mai 2019. URL : http:// journals.openedition.org/ries/3956 ; DOI : 10.4000/ries.3956

Ce document a été généré automatiquement le 1 mai 2019.

(c) Tous droits réservés 


\title{
Multiplicité de modèles en Australie
}

\author{
Michael Berthold
}

1 En Australie, les programmes d'éducation bilingue pour les personnes ne parlant que l'anglais vont de l'enseignement dans une langue étrangère - de trois à cinq heures par semaine - de matières faisant partie du curriculum scolaire (mathématiques, sciences sociales, musique, etc.), à un enseignement dispensé en grande partie, sinon en totalité, dans une langue étrangère. Ce dernier type de programme est appelé «programme d'immersion ».

2 Ces programmes bilingues ont pour objectif d'amener progressivement les étudiants du bilinguisme passif à un bilinguisme actif et, au bout d'un certain temps et dans un climat favorable, au bilinguisme total.

3 Dans cette présentation de l'enseignement bilingue en Australie, nous nous bornerons à décrire sept méthodes actuellement utilisées dans le pays et, à une exception près (les langues aborigènes), appliquées à l'enseignement des langues étrangères et non au maintien de la langue maternelle. Quoique les méthodes d'enseignement bilingue en Australie ne soient pas très connues à l'étranger, le principe même de l'éducation bilingue n'y est pas nouveau puisque l'on en trouve des exemples dès le milieu du XIX ${ }^{e}$ siècle dans des établissements luthériens où certaines matières étaient enseignées en allemand et en anglais.

4 Toutefois, ce type d'enseignement fut interrompu pendant la première guerre mondiale (victime d'une vague de xénophobie), période pendant laquelle l'anglais pour tous était de règle. L'enseignement des langues étrangères était maintenu mais comme un défi intellectuel pour une élite culturelle et non comme un outil de communication.

5 Un changement d'attitudes apparut graduellement, au cours des années soixante-dix, avec le développement d'une politique multiculturelle et les possibilités données aux groupes ethniques de maintenir leur langue maternelle. Des programmes de «maintien linguistique » furent introduits, généralement en dehors des horaires scolaires, par les communautés ethniques. Dans les années quatre-vingts, avec le programme Bayswater, apparurent les premiers programmes d'enseignement bilingue à l'école primaire pour les écoliers dont la langue maternelle était l'anglais. Le plus ancien programme d'immersion 
dans le secondaire fut introduit en 1985 au lycée-collège de Benowa, dans le Queensland. De nombreuses initiatives du même type ont suivi, différenciées selon l'établissement scolaire et la langue enseignée.

6 La situation linguistique en Australie n'est pas du tout comparable à celle de pays bilingues, tels que le Canada, le Pays de Galles ou Hong-Kong. L'Australie n'a qu'une langue officielle, et il n'y a pas de minorité importante ne parlant pas l'anglais. Pour la majorité des étudiants et la communauté en général, l'apprentissage d'une langue autre que l'anglais ne revêt pas une importance significative, l'anglais étant considéré comme suffisant pour la vie quotidienne et professionnelle.

7 En conséquence, quiconque souhaite introduire un programme bilingue en Australie doit tout d'abord surmonter un défi bien plus important que dans les pays officiellement bilingues. Même le choix de la langue à enseigner, choix évident pour le Canada ou le Sénégal par exemple, reste parfois difficile sur ce continent.

Les sept méthodes d'enseignement bilingue présentées ici ainsi que les autres méthodes développées en Australie ont des origines diverses. Certaines écoles ont mis au point des méthodes basées directement sur les modèles canadiens, par exemple à Camberwell (Victoria) où un programme d'immersion partielle et précoce (50\%) en français est utilisé depuis 1991. D'autres modèles ont été développés visant à répondre à des situations spécifiques dans chaque État et pour satisfaire des besoins divers (linguistique, politique, philosophique ou éducatif). Les méthodes décrites dans cet article représentent non seulement un éventail typologique des techniques adoptées, mais aussi constituent les modèles de base sur lesquels s'appuient la plupart, sinon la totalité, des programmes d'éducation bilingue en Australie, à savoir :

- modèle «Bayswater» : primaire ;

- modèle «Telopea Park » : primaire et secondaire ;

- modèle « en escalier » : primaire, secondaire et supérieur ;

- modèle «Benowa » : secondaire ;

- modèle « Mount Scopus » : secondaire ;

- modèle « LACITEP » : supérieur ;

- modèle biculturel « à deux sens » : langues aborigènes.

\section{Le modèle Bayswater ${ }^{1}$}

En 1981, les écoles primaires de Bayswater (dans la région de Melbourne) introduisirent, à titre expérimental, un enseignement bilingue. Dans les classes choisies, les sciences, les arts ou l'éducation physique étaient enseignés exclusivement en allemand, à raison d'une heure par jour. Le programme fut maintenu avec succès et en 1991 tous les élèves de ces écoles suivaient un programme bilingue. La répartition actuelle des leçons basée sur le programme adopté à l'école primaire de Bayswater South est la suivante :

- année préparatoire (Kindergarten) : deux heures et demi par semaine (cinq leçons de trente minutes) ;

- années 1 à 4 : cinq heures par semaine (cinq leçons d'une heure);

- années 5 et $6:$ trois heures par semaine (trois leçons d'une heure).

Le programme Bayswater fut le premier à utiliser une langue étrangère pour l'enseignement d'une autre matière que la langue. On ne saurait toutefois le comparer à un programme d'immersion, même partielle, à cause d'une exposition limitée à la langue 
étrangère. Les écoles primaires de Bayswater (districts Sud et Ouest) ont servi de modèles à la plupart des programmes bilingues dans les écoles primaires du pays. Ils ont été étudiés et suivis dès le début par Michael Clyne et son équipe de l'université de Monash et ont démontré qu'un temps d'immersion, même relativement faible, dans la langue étrangère choisie donne des résultats positifs.

11 Ces programmes bilingues se sont répandus dans les autres États. Par exemple, en Australie occidentale trois programmes similaires existent à l'école primaire en italien (depuis 1991), en français (depuis 1992) à raison de quatre heures par semaine. En Australie méridionale, plusieurs programmes similaires ont été introduits à l'école primaire y compris un programme de huit heures et demi hebdomadaire, en allemand, à l'école de Modbury, ainsi que des programmes en français, italien et espagnol.

\section{Le modèle Telopea Park}

12 Le programme bilingue de Telopea Park à Canberra est le fruit d'un accord entre les gouvernements français et australien ayant conduit à la création d'une école binationale dans laquelle les curricula australien et français sont enseignés tant dans le primaire que dans le secondaire. Le financement est assuré par les deux gouvernements. Les enseignants français sont envoyés par la France pour une courte durée, leur salaire étant payé par le gouvernement français. La première classe du primaire débute par un programme d'immersion à $50 \%$.

13 Ce modèle diffère des autres en ce sens que, d'une part, les curricula des deux pays sont enseignés dans leur langue respective et, d'autre part, il bénéficie d'une participation active des deux gouvernements. L'application de ce modèle restera assez restreinte en Australie. Une tentative similaire a été faite à l'école primaire de Camberwell à Melbourne (1991) mais a été abandonnée pour diverses raisons. Cependant le modèle de Telopea pourrait être appliqué à des initiatives similaires émanant d'autres gouvernements souhaitant introduire ici des programmes bilingues, en anglais et dans leur langue respective.

\section{Le modèle en escalier ${ }^{2}$}

14 Ce modèle s'appuie sur l'utilisation progressive de la langue étrangère amenant les élèves du niveau 1 (monolinguisme) au niveau 4 (bilinguisme). Il n'a pas encore été entièrement expérimenté. Le modèle que nous avons développé vise à amener de manière progressive les enfants au contact de la langue étrangère en augmentant les horaires d'enseignement dans cette langue, pour y inclure des matières autres que la langue elle-même.

Ce programme, qui requiert la coopération des établissements d'enseignement primaire, secondaire et supérieur, a recours à l'enseignement des langues par palier, ce qui permet à un établissement d'y surseoir selon ses contraintes ou ses ressources (voir tableau).

\section{Groupe visé}

Il s'agit des écoles primaires qui souhaitent introduire un programme intégré d'apprentissage d'une langue étrangère, de l'année préparatoire du Kindergarten à la dernière année du primaire, avec continuation dans secondaire. 


\section{Méthode}

Les élèves commencent l'école sans aucune connaissance de la langue étrangère. La méthode débute par un programme d'imprégnation, puis suit les étapes d'apprentissage de la langue cible et peut aller jusqu'à l'enseignement de certaines matières dans cette langue.

Le modèle en escalier : cinq niveaux

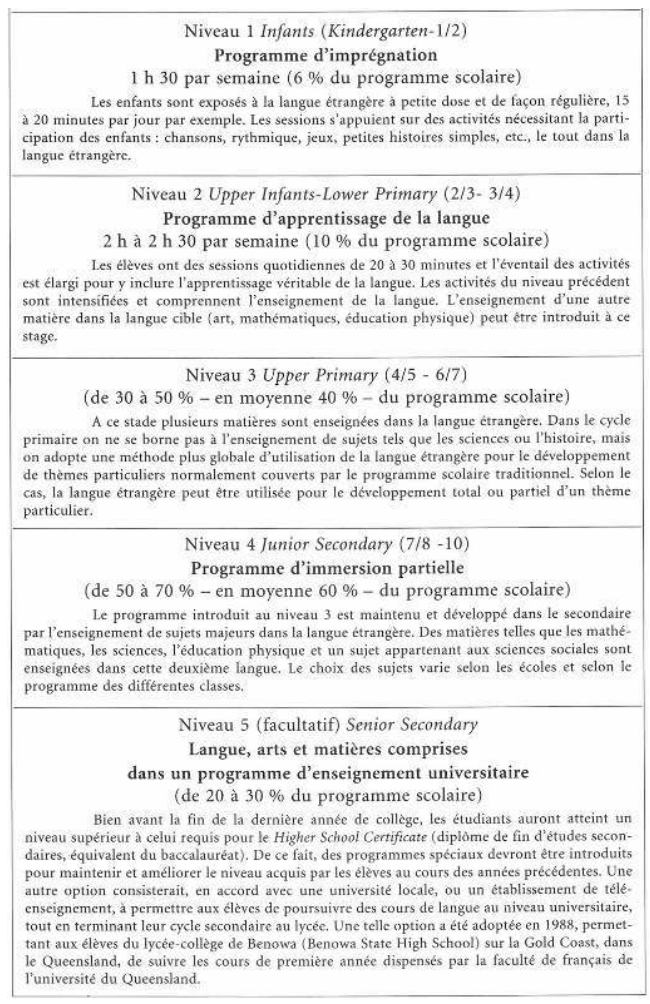

\section{Le modèle Benowa ${ }^{3}$}

Le premier programme d'immersion partielle au niveau secondaire a débuté en 1985 au lycée-collège de Benowa, sur la Gold Coast. L'expérience consistait en une immersion partielle en français. Ce programme, destiné à l'origine à n'être qu'un cours d'enrichissement d'un an réservé aux étudiants les plus doués, évolua en programme de style « immersion partielle tardive » couvrant les trois premières années de la scolarité secondaire. Il a continué à se développer et il comprend maintenant deux classes par année de niveau. Une des caractéristiques qui distingue le modèle Benowa des programmes d'immersion tardifs canadiens est que les enfants qui s'y inscrivent n'ont généralement aucune connaissance en français, alors que les étudiants canadiens bénéficient d'une base en français dès le début des classes élémentaires. Benowa enseignait en français les sujets suivants: mathématiques, sciences, sciences sociales, histoire, éducation physique et français ( $60 \% \mathrm{du}$ programme scolaire hebdomadaire).

Le modèle Benowa fut développé par des professeurs de l'école en réaction à ce qu'ils estimaient être les lacunes des programmes traditionnels de seconde langue et pour 
répondre aux besoins locaux. Il avait ses caractéristiques propres et n'imitait aucun exemple canadien puisqu'il était développé entièrement par l'école, sans connaissance des programmes en cours au Canada et sans aucune influence extérieure. C'est ce modèle qui a été adopté par le ministère de l'éducation du Queensland lors de l'introduction de trois programmes pilotes « officiels» à Mansfield (français, 1991), Kenmore (allemand, 1992) et Park Ridge (indonésien, 1993). Il a servi aussi d'exemple à une école privée de la Gold Coast, The Southport School, qui a suivi cette voie (reprenant aussi une partie importante du matériel d'enseignement) pour développer avec succès son propre programme d'immersion en français.

\section{Le modèle Mount Scopus ${ }^{4}$}

En 1990, à Melbourne, dans l'État du Victoria, l'important collège israélite de Mount Scopus introduit un cycle d'immersion qui concernait $70 \%$ du programme des années 7 et 8 (les deux premières années du secondaire), la langue utilisée étant l'hébreu, dans les sujets suivants : mathématiques, sciences, histoire, géographie, informatique, art dramatique, hébreu et études juives (Munz, 1993: 75). Pendant les années 9 et 10, le programme d'immersion était restreint aux études juives, mais cela représentait en soi $30 \%$ du travail scolaire hebdomadaire enseigné en langue hébraïque. Le modèle de Mount Scopus ressemble davantage aux programmes canadiens traditionnels d'immersion tardive, en ce sens que les élèves avaient été exposés de façon intensive à la seconde langue tout au long du cycle primaire (jusqu'à huit heures d'hébreu par semaine pendant sept ans). Là où il diffère, cependant, c'est dans l'usage d'une langue sans rapport avec l'anglais. De ce fait, il pourrait éventuellement servir de modèle pour des langues comme l'indonésien, l'arabe, le grec, le coréen et même le chinois et le japonais, si l'enseignement de la langue choisie était suffisamment rigoureux au niveau primaire.

21 L'expérience de Mount Scopus a été suivie de près et évaluée par les chercheurs de l'université de Monash et de l'université de Melbourne. Leurs conclusions très positives ont assuré la continuité du programme et nous ont fourni une preuve empirique du succès de l'enseignement d'une langue par immersion en Australie.

\section{Le modèle LACITEP 5}

En 1993, l'université du Queensland Central introduit un programme innovant de formation pour professeurs de japonais, débouchant sur un diplôme en éducation, le Bachelor of Education, et au cours duquel, pendant quatre ans, 50 à $80 \%$ des cours étaient donnés en japonais. LACITEP (Language and Culture Initial Teacher Education: Primary, i.e "Formation pédagogique en langue et culture: enseignement primaire») est un programme ambitieux qui s'est donné pour tâche de préparer les étudiants à un double rôle d'instituteurs primaires et de spécialistes du japonais, en enseignant différentes matières du curriculum dans la langue étrangère. Les étudiants abordant l'enseignement supérieur sont contrôlés afin de s'assurer que leur niveau de japonais à la fin de leurs études secondaires est suffisant pour leur permettre non seulement de faire face à des cours entièrement en japonais, mais encore d'aborder avec succès les différents aspects du contenu, pédagogique ou autre, de leur formation professionnelle. 
23 À la fin de ce programme, les étudiants auront à la fois les qualifications d'enseignants du primaire et celles de spécialistes de la langue japonaise.

\section{Le modèle biculturel « dans les deux sens ${ }^{6}$}

24 Un autre modèle s'est développé en Australie, dans lequel un fort élément culturel est incorporé à l'étude de la langue. Les communautés aborigènes du territoire du Nord, de l'Australie méridionale et occidentale, sont en effet convaincues que la préservation de leur culture revêt autant d'importance que la préservation de la langue, conviction qui a été l'élément moteur dans le développement de ce modèle.

Le système d'éducation des différents États doit donc faire face à un dilemme en ce qui concerne l'éducation aborigène.

Le dilemme est que les aborigènes des régions isolées souhaitent que leurs enfants apprennent les trois R [Reading, (w) Riting and (a) Rithmetic - lire, écrire et compter] tout en étant élevés en aborigènes. Mais si ces enfants obtiennent de bons résultats dans le système scolaire occidental, ce sera vraisemblablement aux dépens de leur culture aborigène, selon la présente structure scolaire. La vision future est que ces écoles peuvent être structurées de façon à ce que les aptitudes nouvelles apprises d'une autre culture s'ajoutent au caractère culturel primaire de l'individu sans le supplanter (Harris, $1990: 1$ ).

La réponse courante à ce problème est d'essayer de développer des programmes qui soient, bien plus que des programmes bilingues, des programmes biculturels « dans les deux sens ». Il faut préserver la langue de la communauté et élever les enfants selon les normes et la culture de la communauté aborigène, mais aussi développer l'instruction et les connaissances en anglais pour permettre aux enfants de se conduire en adultes dans les deux cultures et d'élargir le champ de leurs options. Les communautés ne veulent pas que leurs enfants deviennent des clones de la culture blanche dominante mais au contraire qu'ils maintiennent leur identité tout en restant capables d'évoluer avec une assurance égale dans la société des Blancs.

Bien plus que de travailler à un bilinguisme additionnel, il s'agit de viser à achever un biculturalisme. Une scolarité aborigène « dans les deux sens » comprend des programmes qui, non seulement prépareront les élèves à rester au sein des communautés aborigènes, mais aussi leur permettront de faire face à la culture et à la bureaucratie occidentale lorsque et où ce sera nécessaire. Plusieurs communautés sont en train de développer des modèles qu'ils considèrent comme les plus appropriés à leur situation. Le concept sousjacent, cependant, est que les enfants doivent être élevés dans deux cultures séparées de deux manières différentes et par des tuteurs différents (professeurs, membres de la famille, anciens de la tribu). Ce concept tend à éviter qu'une seule version culturelle soit enseignée en deux langues, la préférence allant à deux cultures enseignées chacune dans leur style distinct et dans la langue appropriée à chaque culture.

Pour qu'un programme aborigène «dans les deux sens » fonctionne, le pouvoir doit se trouver et montrer qu'il se trouve entre les mains des Aborigènes eux-mêmes. Il devra y avoir un net cloisonnement de l'enseignement et de l'apprentissage selon les deux cultures - personnel enseignant, contenu de l'enseignement, langue d'instruction, styles d'enseignement, contexte et choix du moment de l'instruction.

La scolarité «dans les deux sens » n'est pas une chose qui puisse être simplement mise en place par des professeurs non-aborigènes bien intentionnés ou des 
administrateurs de l'extérieur, quoiqu'ils puissent y aider de multiples façons. Par définition, la scolarité « dans les deux sens » est une initiative aborigène interne. La différence principale, quand on la compare avec une éducation bilingue (stratégie positive qui s'en rapproche le plus), telle qu'elle existe actuellement dans quelques écoles aborigènes, est que celle-ci peut être gérée par des éducateurs nonaborigènes travaillant en tandem avec du personnel aborigène; la scolarité « dans les deux sens ", quant à elle, ne peut pas être mise en application de cette manière (Harris, $1990: 19$ ).

\section{Évaluation des programmes} signification de trois facteurs : la durée d'exposition à la langue, la façon naturelle qui accompagne le processus d'acquisition de la seconde langue et l'intégration du programme dans l'ensemble du programme général d'études (Clyne, 1986 : 140). Un autre collègue chercheur précise pour sa part: «Ce que nous avons trouvé indique que le modèle du programme bilingue de Bayswater South est un moyen très efficace pour amener les enfants au bilinguisme tout en présentant aussi des avantages cognitifs et linguistiques pour l'élève. » 

preuves solides de leur efficacité, ainsi :

Le $\mathrm{D}^{\mathrm{r}}$ Peter Cryle, chef de la faculté de français à l'université du Queensland, a écrit que «l'expérience de Benowa est la plus passionnante et la plus productive de toutes celles qui ont été tentées en français dans le Queensland» et que, "sous certains aspects, les meilleurs élèves de l'année 10 sont supérieurs à beaucoup d'étudiants de troisième année à l'université » et encore : «J'ai le ferme espoir que l'Australie verra, dans un avenir proche, des tentatives pour généraliser le modèle Benowa, afin de produire des étudiants naturellement bilingues » (Berthold, 1992 : 18).

Le $\mathrm{D}^{\mathrm{r}}$ Cryle et le personnel de la faculté de français de l'université du Queensland ont testé la compétence des élèves essentiellement dans le domaine de la compréhension et de l'expression orales, en les interrogeant et en évaluant leur degré de compréhension des leçons données en français par leurs professeurs et les membres de l'université.

L'université fut si impressionnée par le niveau de ces apprenants que, lorsque le premier groupe eut fini le programme, à la fin de l'année 10, avec des résultats supérieurs à ceux requis pour le diplôme de fin d'études secondaires (année 12), l'université accepta que certains modules du programme de français de l'université soient enseignés par son personnel pendant les deux dernières années de la scolarité secondaire de ces élèves. Leur niveau avancé de français leur permit donc de suivre des cours de niveau universitaire deux ans avant de quitter le lycée et des équivalences devaient leur être accordées lorsqu'ils s'inscriraient, par la suite, à l'université. Leurs résultats dans les matières choisies furent étonnants, l'un des élèves atteignant même un taux de réussite de $95 \%$ les tests et l'évaluation ayant été conduits par l'université, soucieuse de s'assurer que ses standards étaient maintenus.

\section{Formation pédagogique des professeurs destinés aux programmes d'éducation bilingue}

37 Jusqu'à ces derniers temps, il n'existait pas de programme de formation pédagogique destiné spécifiquement aux professeurs qui avaient (ou qui auraient, le cas échéant) à prendre en charge un enseignement bilingue. Généralement, le personnel de ces programmes était choisi en partant de l'hypothèse que les enseignants, soit instituteurs soit spécialistes de disciplines dans le secondaire, et bilingues de surcroît, seraient du même coup capables d'enseigner avec succès dans des programmes d'éducation bilingue.

Il a été prouvé qu'il s'agissait là d'une hypothèse erronée. Alors que beaucoup parmi ces enseignants se sont montrés à la hauteur de la tâche, un certain nombre d'autres se sont révélés tout à fait inaptes à tenir ce rôle et ont eu un effet négatif sur les progrès des élèves et sur les programmes eux-mêmes.

Récemment deux tentatives ont été faites au niveau universitaire pour essayer de surmonter quelques-unes de ces difficultés. Le programme LACITEP, mentionné plus haut, s'est donné pour tâche, non seulement de former des instituteurs primaires qui soient aussi des spécialistes du japonais mais de les préparer, en outre, à enseigner dans des classes primaires en japonais. La manière même dont ces futurs professeurs sont formés, durant quatre ans, devrait leur assurer une très bonne maîtrise de la méthodologie à employer en fonction de la discipline donnée, ainsi que la maîtrise du 
vocabulaire spécifique et technique, puisqu'ils auront eux-mêmes reçu leur instruction par la méthode d'immersion.

La seule limitation du programme LACITEP est que, actuellement, il ne peut produire qu'un nombre limité de licenciés par an et dans une seule langue, le japonais. Si d'autres institutions d'enseignement supérieur décidaient de suivre cet exemple, peut-être verrions-nous éclore, dans le futur, des programmes équivalents en français, en allemand, en indonésien, etc.

41 L'université de Southern Queensland a adopté une autre approche. Par le biais de l'éducation à distance, elle propose une unité «Enseignement d'une langue par immersion » destinée à tout professeur d'éducation bilingue, en activité ou potentiel, où qu'il se trouve en Australie. L'unité traite de la méthodologie de l'enseignement bilingue à partir d'une base générique, permettant ainsi à tous les enseignants, quelle que soit leur langue d'origine, de participer au programme. L'unité est considérée comme un cours de perfectionnement professionnel, aussi bien en préalable à un emploi que pendant un service effectif, qui couvre non seulement la méthodologie de l'enseignement de type " immersion ", mais aussi le développement des ressources pédagogiques, le bilinguisme, les effets cognitifs du bilinguisme, différents modèles nationaux et internationaux d'éducation bilingue, etc.

Cette présentation synthétique ne vise pas à proposer autre chose qu'une vue générale de ce qui s'est passé jusqu'à ce jour, en Australie, dans le domaine de l'éducation bilingue. Dans l'ensemble, tous les modèles décrits ci-dessus ont été bien accueillis et ont donné des résultats positifs. Il est nécessaire, dans ce pays, de soutenir la diversité des approches en raison du grand nombre de langues qui y sont activement enseignées, soit comme langues étrangères, soit comme langues de communautés (c'est-à-dire parlées par les minorités ethniques australiennes). Il n'existe pas de modèle unique pouvant faire face à toutes les situations. Les établissements scolaires, mais aussi les ministères de l'Éducation, doivent considérer la gamme des modèles dans son ensemble et choisir, puis adapter, ce qu'ils considèrent comme le plus approprié à leurs besoins.

\section{L'enseignement bilingue en France -Gilles Vial}

C'est au lendemain de la dernière guerre mondiale que l'on observe, en France, le premier développement d'un enseignement bilingue, dans sa forme la plus exigeante et la plus complexe, celle de l'enseignement international.

L'installation du quartier général de l'OTAN à Saint-Germain-en-Laye amène en effet à mettre en place au lycée d'Hennemont, en faveur des enfants des militaires stationnés là, des dispositifs d'enseignement novateurs et ambitieux, bilingues et biculturels intégrant des éléments de pédagogie étrangère. La formule française d'enseignement international survit au départ du quartier général et se perfectionne jusqu'au moment où le décret et les arrêtés du 11 mai 1981 la fixent sous les traits qu'on lui connaît aujourd'hui.

L'enseignement bilingue sans ambition internationale proprement dite, encore qu'il semble plus aisé à mettre en place, n'apparaît, paradoxalement, que plus tard. Il 
trouve son origine dans le rapprochement franco-allemand, qui entraîne, en République fédérale d'Allemagne (RFA), la création de classes bilingues francoallemandes et, en France, pour lui faire pendant, les premières sections bilingues, qui sont germanophones, comme en portent témoignage les circulaires du 26 juillet 1971 et du 28 août 1972. Les sections bilingues, par la suite, se diversifient à l'anglais et se multiplient au gré des initiatives rectorales, d'autant plus aisément que le système en est très simple : renforcement horaire et introduction d'une discipline non linguistique (gymnastique, musique) enseignée en langue étrangère, sans aucune sanction de cette scolarité au moment du baccalauréat. Encore maintenant, il se révèle difficile d'identifier toutes les implantations de sections bilingues qui ont subsisté.

C'est la relation franco-allemande, encore, qui inspire la mise au point de deux autres formules d'enseignement bilingue à caractère international (au sens où elles intègrent des éléments non seulement linguistiques, mais aussi culturels et pédagogiques des deux pays) : les lycées franco-allemands (deux établissements en RFA, un en France, à Buc), institués par la convention du 10 février 1972, et les classes de préparation à la délivrance simultanée du baccalauréat et de l'Abitur (sept lycées en France et autant en RFA), institués par l'accord du 27 octobre 1986.

Enfin, dans un passé récent, la pression de la demande d'un enseignement linguistique de haut niveau a amené le ministère de l'Éducation nationale à développer un dispositif d'enseignement bilingue nouveau : les sections européennes et les sections de langue orientale définies par la circulaire n 92234 du 19 août 1992.

Ainsi, en faisant abstraction des systèmes spécifiques mis en place dans le cadre franco-allemand, la France dispose-t-elle principalement de deux dispositifs d'enseignement bilingue distincts, les sections internationales d'une part, les sections européennes, de l'autre.

\section{NOTES}

1. M. Clyne (coord.), An early start: Second Language at Primary School, Melbourne, River Seine Publications, 1986. Fernandez, S., Room for Two, Melbourne, NLLIA, 1992.

2. M.J. Berthold, "The Staircase Model: Language Awareness to Content Teaching", dans: Proceedings of the First Biennial Conference of Australian Association of Language Immersion Teachers, 1993, p. 38-46.

3. M.J. Berthold, «An Australian Experiment in French Immersion », dans: The Canadian Modern language Review, vol. 49, $\mathrm{n}^{\circ} 1,1992$, p. 112-126.

4. M. Munz, «The Implementation of an Immersion Hebrew Programme in a Melbourne Jewish Day School », dans: Proceedings of the First Biennial Conference of Australian Association of Language Immersion Teachers, 1993, p. 75- 81. 
S.C. Loch, T.F. McNamara \& E. Eisikovits, «Late Hebrew immersion at Mount Scopus College, Melbourne: Towards complete Hebrew fluency for Jewish day school students ", dans: Language and Language Education, vol. 2, n 1, 1992, p. 1-29.

5. T. Erben, "Teacher Training Through Immersion", dans: Proceedings of the First Biennial Conference of Australian Association of Language Immersion Teachers, 1993, p. 51-62.

6. S. Harris, Two-Way Aboriginal Schooling, Canberra, Aboriginal Studies Press, 1990.

INDEX

Index géographique : Australie

Mots-clés : enseignement bilingue, formation des enseignants, section bilingue

\section{AUTEUR}

\section{MICHAEL BERTHOLD}

Maître de conférences, université de Southern Queensland, Toowoomba, Australie 